\title{
Evaluation of the Correlation Coefficient as a Prognostic Indicator for Electromechanical Servomechanism Failures
}

\author{
Matteo D. L. Dalla Vedova ${ }^{1}$, Paolo Maggiore ${ }^{2}$ Lorenzo Pace $^{3}$ and Alessio Desando ${ }^{4}$ \\ ${ }^{1,2,3,4}$ Politecnico di Torino, Torino, 10129, Italy \\ matteo.dallavedova@polito.it \\ paolo.maggiore@polito.it \\ lorenzo.pace@polito.it \\ alessio.desando@polito.it
}

\begin{abstract}
In order to identify incipient failures due to a progressive wear of a primary flight command electromechanical actuator, several approaches could be employed; the choice of the best ones is driven by the efficacy shown in fault detection/identification, since not all the algorithms might be useful for the proposed purpose. In other words, some of them could be suitable only for certain applications while they could not give useful results for others. Developing a fault detection algorithm able to identify the precursors of the abovementioned electromechanical actuator (EMA) failure and its degradation pattern is thus beneficial for anticipating the incoming malfunction and alerting the maintenance crew such to properly schedule the servomechanism replacement. The research presented in the paper was focused to develop a fault detection/identification technique, able to identify symptoms alerting that an EMA component is degrading and will eventually exhibit an anomalous behavior, and to evaluate its potential use as prognostic indicator for the considered progressive faults (i.e. frictions and mechanical backlash acting on transmission, stator coil short circuit, rotor static eccentricity). To this purpose, an innovative model based fault detection technique has been developed merging several information achieved by means of Fast Fourier Transform (FFT) analysis and proper "failure precursors" (calculated by comparing the actual EMA responses with the expected ones). To assess the performance of the proposed technique, an appropriate simulation test environment was developed: the results showed an adequate robustness and confidence was gained in the ability to early identify an eventual EMA malfunctioning with low risk of false alarms or missed failures.
\end{abstract}

Matteo D. L. Dalla Vedova et al. This is an open-access article distributed under the terms of the Creative Commons Attribution 3.0 United States License, which permits unrestricted use, distribution, and reproduction in any medium, provided the original author and source are credited.

\section{INTRODUCTION}

As defined by Vachtsevanos, Lewis, Roemer, Hess, \& Wu (2006), the purpose of prognostics is to predict accurately the Remaining Useful Life (RUL) of a failing component or subsystem. In fact, especially in aeronautics but also in many other technological fields, the development of a prognostics health management (PHM) based fault-tolerant control architecture can increase safety and reliability by detecting and accommodating impending failures thereby minimizing the occurrence of unexpected, costly and possibly life-threatening mission failures; reduce unnecessary maintenance actions; and extend system availability / reliability. The advantages gained by means of PHM strategies are evident comparing the features of a system developed according to this discipline with the ones of a classical design. The primary flight controls are a critical feature of the aircraft system and are therefore designed with a conservative safe-life approach, which imposes to replace the related components subsequently to a certain number of flight hours (or operating cycles). This approach fails in the detection of possible initial flaws, due to the manufacturing process, that could generate a sudden failure, which could compromise the safety of the aircraft. Moreover, this design criterion merely requires the component replacement, regardless of its effective status or capability to operate still correctly, with the related inefficiencies and additional costs.

Instead, in a system suitably designed by taking into account the PHM strategies, the failures could be managed in a more proper way, obtaining the following advantages:

1. lower operating costs;

2. less maintenance interventions are required;

3. lower number of redundancies installed on board aircraft;

4. aircraft safety and reliability are improved; 
5. any maintenance work can be planned appropriately optimizing the necessary actions (limiting downtime and related costs and allowing a more effective organization of the maintenance and management of spare parts warehouses) and limiting the logistical difficulties resulting from the manifestation of the fault.

The research presented in this paper was focused on two main objectives. The first is the proposal of a numerical algorithm capable to simulate the dynamic behavior of a typical electromechanical servomechanism affected by one or multiple progressive faults. The developed numerical model takes into account dry friction, backlash, coil short circuit and rotor static eccentricity. The second consists in the development of an innovative fault detection/evaluation technique able to identify failure precursors (alerting that the system is degrading) and to evaluate the corresponding damage entity. The progressive degradation of a system component, indeed, does not initially create an unacceptable behavior, but only eventually leads to a condition in which the whole actuation system operation could be compromised. In order to fulfill these two objectives, an innovative model-based fault detection and identification technique has been developed merging several information achieved by means of FFT analysis and proper failure precursors, i.e. system parameters whose variations could be associated with specific impending failure (Vichare \& Pecht 2006). This technique was tested by means of numerical simulations on a typical aircraft primary command EMA, modeled in the MATLAB Simulink ${ }^{\circledR}$ simulation environment. The proposed method showed an adequate robustness, and more confidence was gained in the ability to early identify the malfunctioning, with low risk of false alarms or missed failures.

The present work started with an extensive literature review, focused both on the most common EMA fault modalities and propagation models and on the techniques and algorithms allowing their detection and evaluation. Then, the numerical models, implemented in MATLAB Simulink $®$ and utilized to analyze the fault modalities, were described. The first model, described in Section 3, represents the typical electromechanical servomechanism, and allows simulating the effects due to the four different types of progressive faults previously mentioned. This model was coupled to the second one (see Section 4), which represents the same EMA working in ideal conditions, i.e. unaffected by any failure modality. The idea was to detect the effects due to progressive faults by comparing the performance of the former with the response of the latter, utilized as a monitoring model. By means of proper algorithms, the obtained results could be later used to timely identify the faults and evaluate their magnitudes. In Section 5 , the numerical modeling of the above-mentioned faults was discussed in detail, such as their effects on the system and the parameters allowing each fault to be detected.
Finally, Section 6 was focused on generating several feature maps for each fault modality with the previously found parameters. Then, the obtained maps were utilized for the successful evaluation of the damage level affecting the EMA.

\section{BACKGROUND}

(a) EMA Faults and Degradations

EMAs have only recently been applied in aeronautics. Therefore, the cumulated flight hours or on-board installations of such units are not so much to achieve a reliable statistics about the most recurring failures. Gökdere, Chiu, Keller \& Vian (2005) show that it is possible to discern between four main categories of failures:

1. mechanical or structural failures;

2. BLDC motor failures;

3. electronics failures;

4. sensor failures.

The present work has been mainly focused on the effects of mechanical failures due to progressive wear, which causes an increase of backlash and friction, and on two typical BLDC motor faults: the coil short-circuits ad the bearing wear generating rotor static eccentricity.

Sensor and electrical components failures are not less important than the other ones and their faults are often characterized by rather fast temporal evolutions. However, considering suitable time scales, it is possible to evaluate precursors that can be used to take action (Ginart, Brown, Kalgren \& Roemer, 2010). Nevertheless, they are not considered in this work because their time scales result often much shorter than the ones related to above mentioned progressive faults. It is the intention of the authors to study these types of failure in a next work.

Dry friction phenomena occur when two surfaces are in relative motion: as the friction coefficient increases due to wear, reaction torque becomes higher and the motor must provide higher torques to actuate the control surface. As shown by Borello, Maggiore, Villero and Dalla Vedova (2010), increased dry friction, while still not causing the seizure of the entire system, reduces the servomechanism accuracy and, sometimes, influences the system dynamic response generating unexpected behaviors, such as stick-slip or limit cycles.

The mechanical wear could also generate backlash in EMA moving parts such as gears, hinges, bearings and especially screw actuators. These backlashes, acting on the elements of the mechanical transmission, reduce the EMA accuracy and can lead to problems of stiffness and controllability of the whole actuator, as shown by Borello and Dalla Vedova (2006). 
BLDC motor faults are mainly seen as progressive stator coil short-circuits or bearing wear generating rotor static eccentricity. As shown by Shashidhara \& Raju (2013), there is a consensus that 35-40 \% of induction motor breakdowns could be attributed to the stator winding insulation. Despite of the causes, stator winding-related breakdown could be segregated into five types: turn-to-turn, coil-to-coil, line-toground, line-to-line, and open-circuit faults. Among the five fault modes, turn-to-turn faults (stator turn fault) is supposedly the most challenging one as the other forms of breakdown are generally the result of turn faults. Jeong \& Hur (2013) state that a significant part of a stator winding-connected failures is induced by the insulation failures in multiple turns of a stator coil within one phase. This kind of fault, known as a "stator turn fault", usually starts between a few turns belonging to the same phase coil. Since into short-circuited coils the voltage remains the same and the resistance decreases, a high circulating current arises, generating a localized heating in conductor: this heating favors the extension of the fault to adjacent coils. If this kind of fault is not promptly detected, it could propagate and generate phase-phase or phase-neutral damages (Jeong \& Hur, 2013).

The static eccentricity of a rotating body consists in a misalignment between the rotor rotation axis and the stator axis of symmetry. This misalignment is mainly due to tolerances and imperfections during motor construction or to a gradual increase of wear of the rotor shaft bearings. According to Akar, Taskin, Seker and Cankaya (2010), this displacement of symmetrical axes could be classified into static, dynamic and mixed eccentricities; in this work, only the first type of eccentricity will be discussed. One can notice that, in case of static eccentricity fault, the motor having more than one polar couple generates a periodically variable magnetic flux, since the air gap varies during its $360^{\circ}$ degrees turn (Bruzzese \& Joksimovic, 2011).

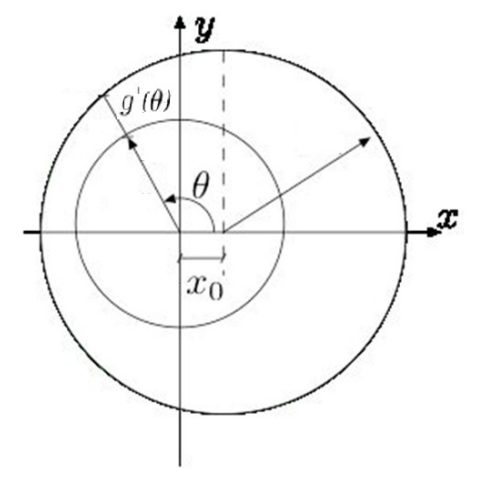

Figure 1. Reference system for the definition of air gap.

In case of static eccentricity, the air gap changes during a spin of the rotor (Figure 1) and its behavior could be represented by the function:

$$
g^{\prime}(\vartheta)=g_{0}+x_{0} \cos (\vartheta)
$$

where $g_{0}$ is the clearance between stator and rotor (without misalignment) and the second term represents the variation of the air gap with $\vartheta$ related to the misalignment $x_{0}$. In terms of motor performances, provided torque is lower than in nominal conditions, whilst spectral analysis reveals subharmonics increasing for higher eccentricities (Kim, Kim, \& Hur, 2012).

\section{(b) Fault Detection/Identification Algorithms}

The fault detection/identification could be achieved by means of a proper algorithm (typically applied to a numerical model) able to detect the progressive faults and predict their evolution. This fact underlines a limit of prognostics: it could predict only faults presenting a gradual growth and it is not able to detect sudden failures. Prognostics algorithms can have several complexity levels, from the simplest based on heuristic criteria to the most complex involving physical fault models. Developing a prognostic algorithm able to identify the precursors of an EMA failure and its degradation pattern is thus beneficial for anticipating the incoming failure and alerting the maintenance crew such to properly schedule the EMA replacement. This avoids a servomechanism failure in service, thereby ensuring improved equipment availability and minimizing the impacts onto the logistic line. To this effect, a model based fault detection/evaluation technique was developed that fuses several information obtained by comparing actual with expected responses of the EMA to recognize a degradation and estimate the remaining useful life. The choice of the best algorithms able to detect and evaluate a particular kind of incipient failure is driven by their ability to detect the fault itself, so proper tests are needed. The proposed algorithm is based upon:

1. Fourier spectral analysis (by means of Fast Fourier Transform, or FFT);

\section{Correlation coefficient.}

The Fourier Transform (FT) is a mathematical instrument, based upon the theory of Fourier series, which has many applications in physics and engineering (Welch, 1967). Fourier Transform of a function $f(t)$ is often calculated by means of the Discrete Fourier Transform (called DFT). Unlike the typical FT, the DFT requires as input a discrete function; this restrains the DFT to the analysis of a function on a limited and discrete domain. It must be noted that the input values of DFT are finite sequences of real or complex numbers, feature that makes it ideal for data processing on electronic calculators; in particular, this method is employed to analyse the frequencies composing a certain numerical signal by means of proper algorithms constituting the FFT (as shown by Cardona, Lerusse \& Géradin, 1998). In order to achieve the spectral analysis of the dynamic response of the actuation system to a given command, a dedicated numerical algorithm (based upon the FFT MATLAB implementation) has been designed. 
The other instrument used to detect progressive faults or wear conditions is the correlation coefficient $\mathrm{C}$. This coefficient, as proposed by Borello, Dalla Vedova, Jacazio and Sorli, M. (2009) and Dalla Vedova et al. (2010), is defined as:

$$
C=\frac{\int_{0}^{T} x_{T} x_{M} d t}{\int_{0}^{T} x_{T}^{2} d t}
$$

where $x_{T}$ is the set of observed data and $x_{M}$ is the theoretical data: in this work, they are respectively the results of the model that simulates the actual system and the data from the monitoring model. The data considered in the two vectors, depending on the case, could concern positions, velocities or other physical magnitudes of the system. The data representing the dynamic response of the actual system (fault sensitive) are compared with the results provided by the monitoring system (that simulates ideal conditions, since no progressive faults are considered): the more the fault is considerable, the more the results obtained from the simulated actual system differ from the theoretical data. Then, the analysis is aimed to identify a certain physical magnitude, which is sensitive to the occurring fault, and to correlate its variations to the fault growth. In order to be useful for the prognostic analysis, this correlation should have a monotonic trend as the corresponding fault increases and be easily detectable. To this purpose, the authors have defined another dedicated numerical algorithm (developed in MATLAB environment) implementation (2).

\section{ACtUATION System NuMERICAL MODEL}

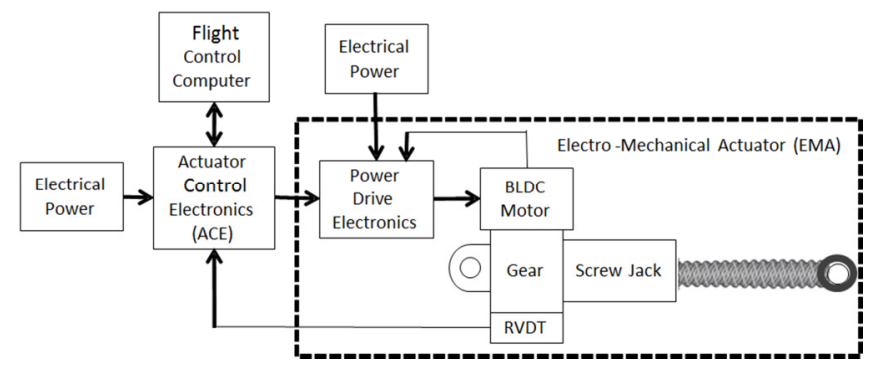

Figure 2. EMA scheme.

As shown in Figure 2, a typical electromechanical actuator used in a primary flight control system is composed by:

1. an actuator control electronics (ACE) that closes the feedback loop comparing the commanded position (FBW) with the actual one, elaborates the corrective actions and generates the reference current $\left(I_{\text {ref }}\right)$;

2. a Power Drive Electronics (PDE) that regulates the three-phase electrical power;

3. an electrical motor, often BLDC type;
4. a gear reducer having the function to decrease the motor (angular) velocity (called RPM) and increase its torque at values suitable for the user ${ }^{1}$;

5. a system that transforms rotary motion into linear motion: ball screws or roller screws are usually preferred to acme screws since they, having a higher efficiency, perform the conversion with lower friction;

6. a network of sensors used to close the feedback rings (current, angular speed and position) that control the whole actuation system (called RVDT).

As previously stated, the primary goal of the research is the proposal of a technique able to identify symptoms alerting that an EMA is degrading: therefore, in order to assess the robustness of this technique, a suitable simulation test environment has been developed. The proposed numerical model, reported in Figure 3, is consistent with the EMA architecture shown in Figure 2 and has been implemented in the MATLAB/Simulink ${ }^{\circledR}$ environment. The model is also able to simulate the effects due to conversion from analogic to digital of the feedback signals (ADC), electrical noise acting on the signal lines and position transducers affected by electrical offset. It is composed by six different subsystems that will be briefly described in the followings.

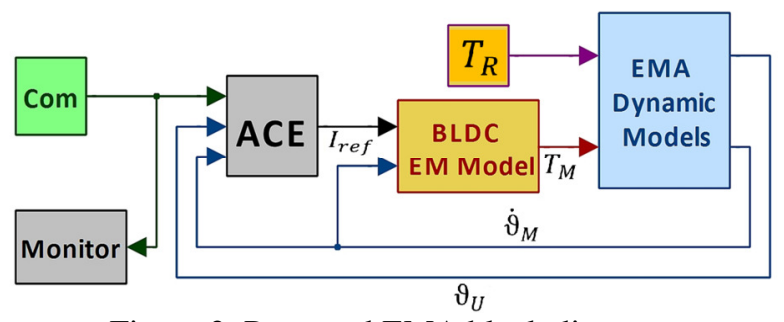

Figure 3. Proposed EMA block diagram.

(a) Com

An input block that generates the different position commands. It is possible to provide several types of input command, such as ramp, step or sinusoid.

\section{(b) ACE}

A subsystem that, as shown by Todić, Miloš and Pavišić (2013), simulates the Actuator Control Electronics unit closing the feedback loops and generating as output the reference current $I_{\text {ref }}$.

\section{(c) BLDC EM Model}

This subsystem simulates a brushless direct current (BLDC) electric motor: It consists of the power drive electronics and the trapezoidal BLDC electromagnetic model, that evaluates the torque developed by the electrical motor as a function of the voltages generated by the three-phase electrical power regulator.

1 The RPM or torque variations are obviously related to the gear ratio of the mechanical reducer. The output torque (downstream the reducer) is also affected by efficiency of the mechanical transmission. 


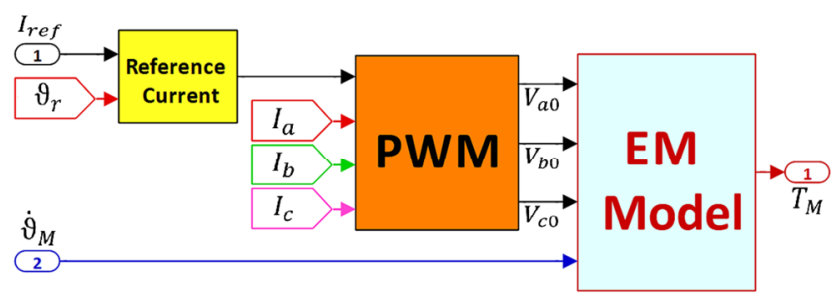

Figure 4. BLDC EM Model block diagram.

Figure 4 shows the numerical simulation algorithm that implements the BLDC motor electromagnetic model. It is composed by three blocks representing the reference current generator, the three-phase pulse-width modulation (PWM) inverter system and the BLDC motor electromagnetic model. This model has been developed according to the mathematical models and the assumption proposed by Çunkas and Aydoğdu(2010) and Halvaei Niasar, Moghbelli and Vahedi (2009).

The trapezoidal counter-electromotive force (or back-EMF) and the electrical current waveforms of the three-phase BLDC motor, evolving as a function of rotor position $\left(\vartheta_{r}\right)$, are shown in Figure 5.

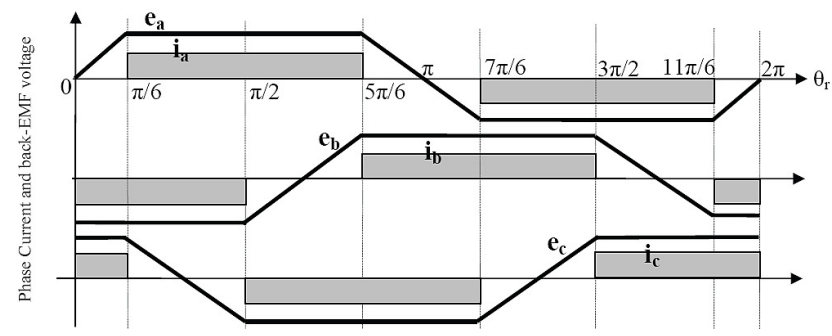

Figure 5. Phase back-EMF and current waveforms of a three-phase BLDC motor.

The abovementioned trapezoidal back-EMFs $e_{a}, e_{b}$, and $e_{c}$ can be expressed as a function of the corresponding electric rotor position as reported in (3).

The Reference Current generator determines the motor reference phase currents $\left(I_{r e f, a}, I_{r e f, b}, I_{r e f, c}\right)$ by considering the reference current amplitude $\left(I_{\text {ref }}\right)$, which is calculated depending on the rotor position; in line with (3), the phase currents are modeled as shown in Table 1.

As proposed by Hua and Zhiyong (2008), the PWM current control block simulates the inverter behavior comparing the reference phase currents $\left(I_{r e f, a}, I_{r e f, b}, I_{r e f, c}\right)$ with the motor's actual phase currents $\left(I_{a}, I_{b}, I_{c}\right)$. Indeed, this block diagram does not implement the structure and the real operation of the three-phase PWM inverter. Its behavior is simulated by means of a relay block, having proper thresholds (set by user), for each phase. The output of this subsystem is a rotating voltage vector having three 120degree displacement components, one for each phase, representing the corresponding phase voltages $V_{a 0}, V_{b 0}$ and $V_{c 0}$.

$$
\begin{aligned}
& e_{a}=\left[\begin{array}{cc}
\left(\frac{6 E}{\pi}\right) \vartheta_{\tau}\left(0<\vartheta_{\tau}<\frac{\pi}{6}\right) \\
E & \left(\frac{\pi}{6}<\vartheta_{\tau}<\frac{5 \pi}{6}\right) \\
-\left(\frac{6 E}{\pi}\right) \vartheta_{\tau}+6 E & \left(\frac{5 \pi}{6}<\vartheta_{\tau}<\frac{7 \pi}{6}\right) \\
-E & \left(\frac{7 \pi}{6}<\vartheta_{\tau}<\frac{11 \pi}{6}\right) \\
\left(\frac{6 E}{\pi}\right) \vartheta_{\tau}-12 E & \left(\frac{11 \pi}{6}<\vartheta_{\tau}<2 \pi\right)
\end{array}\right] \\
& e_{b}=\left[\begin{array}{cc}
-E & \left(0<\vartheta_{\tau}<\frac{\pi}{2}\right) \\
\left(\frac{6 E}{\pi}\right) \vartheta_{\tau}-4 E & \left(\frac{\pi}{2}<\vartheta_{\tau}<\frac{5 \pi}{6}\right) \\
E & \left(\frac{5 \pi}{6}<\vartheta_{\tau}<\frac{9 \pi}{6}\right) \\
-\left(\frac{6 E}{\pi}\right) \vartheta_{\tau}+10 E & \left(\frac{9 \pi}{6}<\vartheta_{\tau}<\frac{11 \pi}{6}\right) \\
E & \left(\frac{11 \pi}{6}<\vartheta_{\tau}<2 \pi\right) \\
E & \left(0<\vartheta_{\tau}<\frac{\pi}{6}\right) \\
-\left(\frac{6 E}{\pi}\right) \vartheta_{\tau}+2 E & \left(\frac{\pi}{6}<\vartheta_{\tau}<\frac{\pi}{2}\right) \\
-E & \left(\frac{\pi}{2}<\vartheta_{\tau}<\frac{7 \pi}{6}\right) \\
\left(\frac{6 E}{\pi}\right) \vartheta_{\tau}-8 E & \left(\frac{7 \pi}{6}<\vartheta_{\tau}<\frac{9 \pi}{6}\right) \\
E & \left(\frac{9 \pi}{6}<\vartheta_{\tau}<2 \pi\right)
\end{array}\right]
\end{aligned}
$$

As happens for the $I_{r e f}$ calculation, at a same instant a phase has a positive value, another has a negative value having the same modulus of the positive one and the remaining one must be null (the proposed model realizes this last statement only on a mean value).

The EM Model calculates the three-phase currents $\left(I_{a}, I_{b}\right.$, $I_{c}$ ) and the developed mechanical torque $T_{M}$ as a function of the PWM three phase voltages $\left(V_{a 0}, V_{b 0}, V_{c 0}\right)$ and the effective rotor velocity $\dot{\vartheta}_{M}$. The considered BLDC motor has a three-phase winding topology with star connection: it has three resistive $(R)$ - inductive $(L)$ branches on which a back-EMF ${ }^{2}$ acts.

Table 1 Reference Currents of BLDC Motor

\begin{tabular}{cccc}
\hline $\begin{array}{c}\text { Rotor Position } \\
\text { (Degree) }\end{array}$ & \multicolumn{3}{c}{$\begin{array}{c}\text { Reference Currents } \\
\text { (A) }\end{array}$} \\
\hline & $I_{\text {ref,a }}$ & $I_{\text {ref }, b}$ & $I_{\text {ref }, c}$ \\
\hline $0-30$ & 0 & $-I_{\max }$ & $I_{\max }$ \\
$30-90$ & $I_{\max }$ & $-I_{\max }$ & 0 \\
$90-150$ & $I_{\max }$ & 0 & $-I_{\max }$ \\
$150-210$ & 0 & $I_{\max }$ & $-I_{\max }$ \\
$210-270$ & $-I_{\max }$ & $I_{\max }$ & 0 \\
$270-330$ & $-I_{\max }$ & 0 & $I_{\max }$ \\
$330-360$ & 0 & $-I_{\max }$ & $I_{\max }$ \\
\hline
\end{tabular}

2 In nominal conditions (no fault considered) the back-EMF acting e.g. on the phase "a" is a function of the rotor position $\vartheta_{M}$ having the amplitude of $e_{a}=k_{e} \cdot \dot{\vartheta}_{M}$, that $k_{e}$ is back-EMF constant of the considered phase. In case of BLDC fault (such as coil short-circuits or static eccentricity), the back-EMF constants may be suitably modified by means of three functions $f(u)$ (one for each motor phase) properly designed in order to simulate the effects of these faults. 
According to Halvaei Niasar et al. (2009), the back-EMF phase voltages are implemented by using Simulink look-up table functions. It must be noted that the three back-EMF constants $k_{e, i}$ (one for each of the three branches) may also take into account some possible BLDC faults (like partial coil short circuit or rotor static eccentricity) by modifying the parameters of the function $f(u)$ : these values, multiplied by the effective rotor velocity $\dot{\vartheta}_{M}$, provide the corresponding real back-EMF values. In order to calculate the value of the three phase currents $\left(I_{a}, I_{b}, I_{c}\right)$, it is necessary to evaluate the differential voltage acting on each phase. As shown in Figure 6, the proposed model considers the three-phase currents in terms of the line-to-line voltages (i.e. the differential voltage between two of the three phases). These differential voltages $\left(\Delta V_{a b}, \Delta V_{a c}, \Delta V_{b c}\right)$ are calculated as difference between the corresponding line-toline values of voltage $\left(V_{a b}, V_{a c}, V_{b c}\right)$ and back-EMF $\left(e_{a b}\right.$, $\left.e_{a c}, e_{b c}\right)$.

Once the line-to-line differential voltages have been calculated, it is possible to derive the three phase currents $\left(I_{a}, I_{b}, I_{c}\right)$ according to the relations reported in (4) and (5):

$$
\begin{gathered}
{\left[\begin{array}{l}
I_{a}=I_{1}-I_{3} \\
I_{b}=I_{2}-I_{1} \\
I_{c}=I_{3}-I_{2}
\end{array}\right]} \\
{\left[\begin{array}{l}
\Delta V_{a b}=V_{a b}-e_{a b}=R_{L L} I_{1}+L_{L L} \frac{d I_{1}}{d t} \\
\Delta V_{b c}=V_{b c}-e_{b c}=R_{L L} I_{2}+L_{L L} \frac{d I_{2}}{d t} \\
\Delta V_{c a}=V_{c a}-e_{c a}=R_{L L} I_{3}+L_{L L} \frac{d I_{3}}{d t}
\end{array}\right]}
\end{gathered}
$$

in which resistance $L_{L L}$ and inductance $R_{L L}$ values are twice those of the phase, because calculations involve two branches; the three phase currents are obtained as simple difference between the corresponding line-to-line currents.

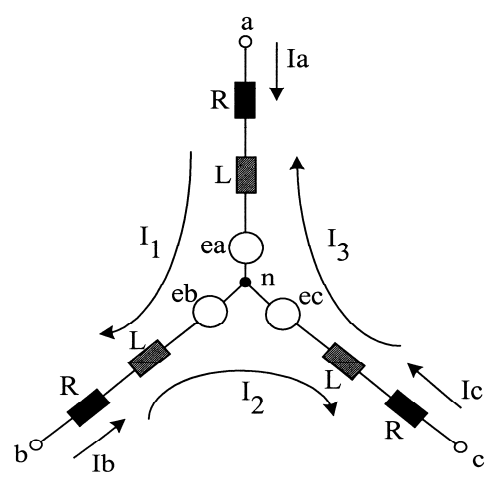

Figure 6. Schematic of voltage and current parameters in three-phase BLDC motor.

Since phase currents are known, total motor torque can be computed; the sum of the three phase currents, multiplied by their respective back-EMF constants $k_{e, i}$ gives the corresponding value of the total motor torque $T_{M}$.
However, this value is limited by means of a Simulink Saturation block in order to take in account the operating limitations of the real system, due to the characteristics of the different components constituting the actuator himself.

It must be noted that, in order to validate the just illustrated numerical model, the dynamic response developed by the aforesaid system under certain operating conditions (control input, boundary conditions and entities of different faults) was compared with data obtained from literature. In particular, the back-EMF and phase currents waveforms, related to different values of the rotor angular velocity, and the dynamic responses of the BLDC motor, caused by various command inputs, have been compared with corresponding cases available in literature (Lee \& Ehsani, 2003), highlighting a satisfactory compliance between simulations and literature data.

\section{(d) EMA Dynamic Model}

This subsystem models the EMA mechanical behavior, by means of a 2-degree of freedom dynamical system. As shown in Figure 7, it consists of two non-linear second order dynamic models linked together by means of a stiffness. The first dynamic system is related to the group including motor and gears (BLDC Motor block), while the second one represents the final user (USER block), i.e. the aircraft command surface controlled by the EMA. They are linked together through an instantaneous model simulating the elastic reaction $T_{\text {reaz }}$ due to shaft stiffness, the Mechanical Transmission block.

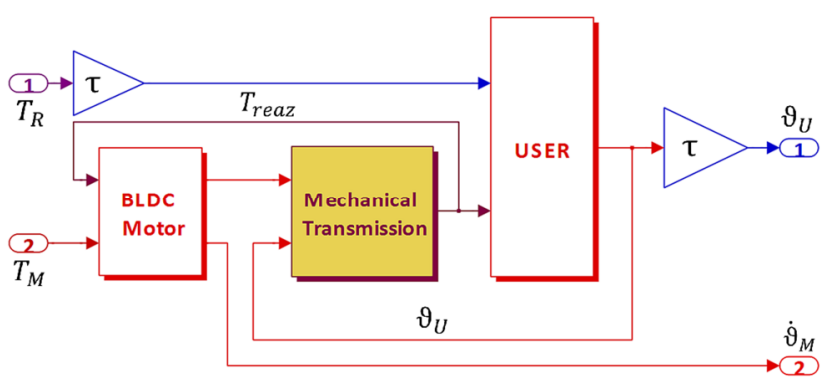

Figure 7: EMA Dynamic Models block diagram.

This type of simulation algorithm, widely explained by Borello and Dalla Vedova (2012), is also able to simulate the effects of the dry friction forces developed in rotor bearings, gear reducer, hinges and screw actuators; in particular, the frictional torque is calculated by means of the numerical model proposed by Borello et al. (2009).

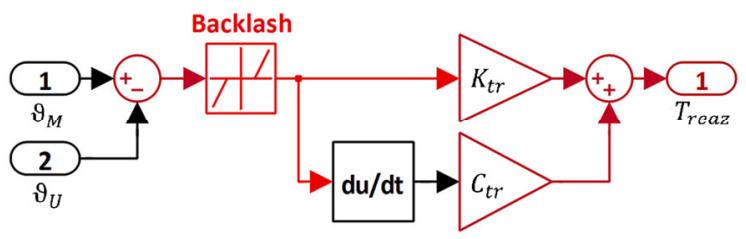

Figure 8: Mechanical Transmission block diagram. 


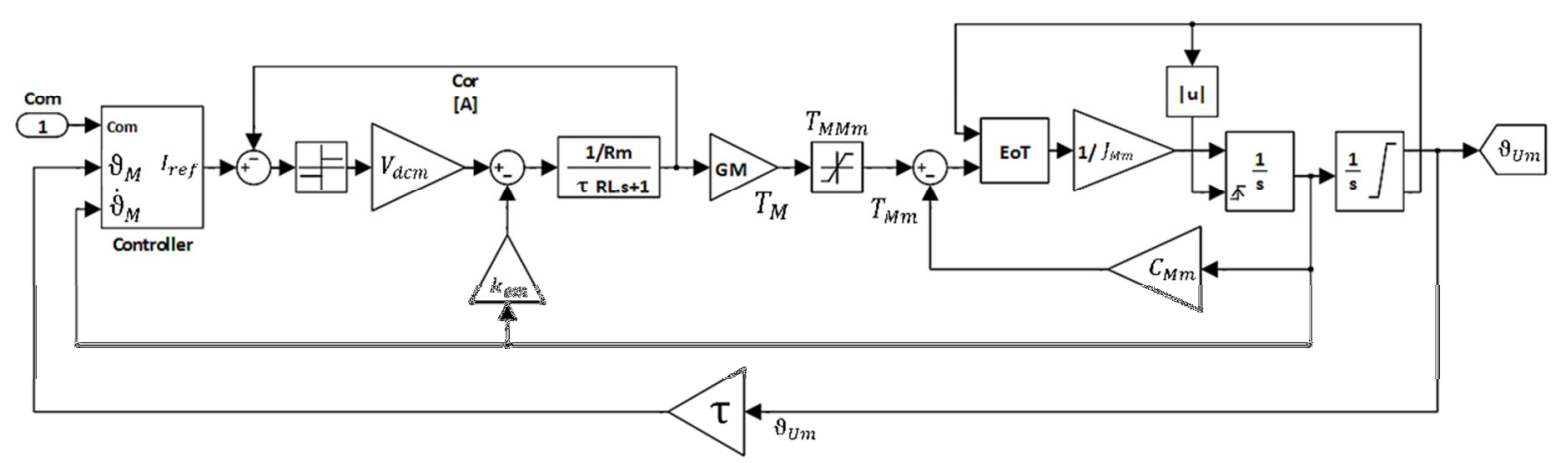

Figure 9: Monitoring Model block diagram.

The Mechanical Transmission subsystem shown in Figure 8 simulates the behavior of the transmission shaft that links together the gear-motor assembly and the final user, calculating the corresponding reaction torque $T_{\text {reaz }}$. This value is modeled as a function of $\vartheta_{M}, \vartheta_{U}$ (respectively motor and user position) and the transmission shaft stiffness $K_{t r}$, and takes into account the effects of the mechanical backlashes.

\section{(e) TR}

Simulates the aerodynamic torque, acting on the moving surface controlled by the actuator.

(f) Monitor

Represents an ideal EMA: it is used as a monitoring system. It is discussed more in detail in the following section.

\section{RELATED MONITORING MODEL}

The above Simulink model, as explained in the previous section, is able to simulate the dynamic behavior of an actual electromechanical servomechanism. It takes into account the effects due to command inputs, environmental boundary conditions and several faults. So it allows simulating the dynamic response of the real system, in order to evaluate the effects due to different faults and, consequently, to develop different diagnostic and prognostic monitoring strategies. In order to conceive a smart system able to identify and evaluate the progressive faults, it is necessary to compare its dynamic behavior with the response provided by an ideal system operating in nominal conditions (i.e. the effects due to faults are neglected). To this purpose, a second numerical model, dedicated to monitoring operations, has been developed. As shown in Figure 9, the Monitoring Model Controller represents a simplified and more compact version of the proposed EMA numerical model, having the same logical and functional structure. This model, with respect to the detailed one, provides similar, although less detailed, performance, with the advantage to reduce computational effort and time. The Controller calculates the output reference current $I_{\text {ref }}$ as a function of the motor angular position $\vartheta_{M}$, the motor angular velocity $\dot{\vartheta}_{M}$ and the commanded position Com.
In order to simplify the electromagnetic numerical model, the three-phase BLDC motor has been modeled as an equivalent single-phase electromagnetic motor and the driving torque $T_{M}$ is directly obtained multiplying the current by a torque constant $G_{M}$.

The difference between reference $\left(I_{\text {ref }}\right)$ and actual currents (Cor) enters a SIGN block that returns the corresponding phase supply voltage $\pm V_{d c m}$ (respectively, when reference current is higher than actual current or vice versa). These values, decreased of back-EMF, allow calculating (by means of a transfer function modeling the resistiveinductive circuit) the actual phase current Cor used in feedback for motor torque computation $T_{M}$. A saturation block is provided to take into account the corresponding torque limits. In the aim to simplify the actuator mechanical model, the gear/motor-user assembly has been degraded to a 1 degree of freedom (d.o.f.) non-linear second order dynamic system. All the effects due to system inertias, transmission shaft stiffness, backlashes and friction torques have been neglected. Moreover, the inertial and viscous terms have been reduced to the same shaft.

\section{NON-LINEAR BEHAVIORS AND FAULTS EFFECTS}

In order to recognize the effects produced by a fault on the dynamic behavior of the considered actuation system, the dynamic responses generated under such conditions need to be compared with those recorded in nominal conditions (NC). Nominal conditions are set considering the system under proper values of dry friction torque and mechanical backlash, while phase short circuit and rotor static eccentricity are not occurring.

\section{(a) Non-linear Behaviors in Nominal Conditions}

Firstly, the proposed EMA model has been tested with several simulations in nominal conditions (NC). The aim was to verify that the proposed numerical model is capable to replicate the most common non-linear phenomena occurring during the operating conditions of a real EMA. These tests have been performed considering many types of input command. 
A step command input (Figure 10) generates a dynamical response that, in NC (having proper values of dry friction torque and mechanical backlash and neglecting any phase short circuit or rotor static eccentricity), it allows to evaluate the dynamic response in transient (e.g. possible overshoots and related dynamic attenuation, settling time, steady state error). Another feature noticeable in the same graph is the presence of the saturations and the position errors due to frictions. These non-linear effects can be visible since the authors' model integrates the dry friction algorithm in a dynamic system able to take into account also the hard stops effects and their mutual interactions. This model proofs to be capable to discern between static and dynamic friction conditions and to evaluate their effects on the system.
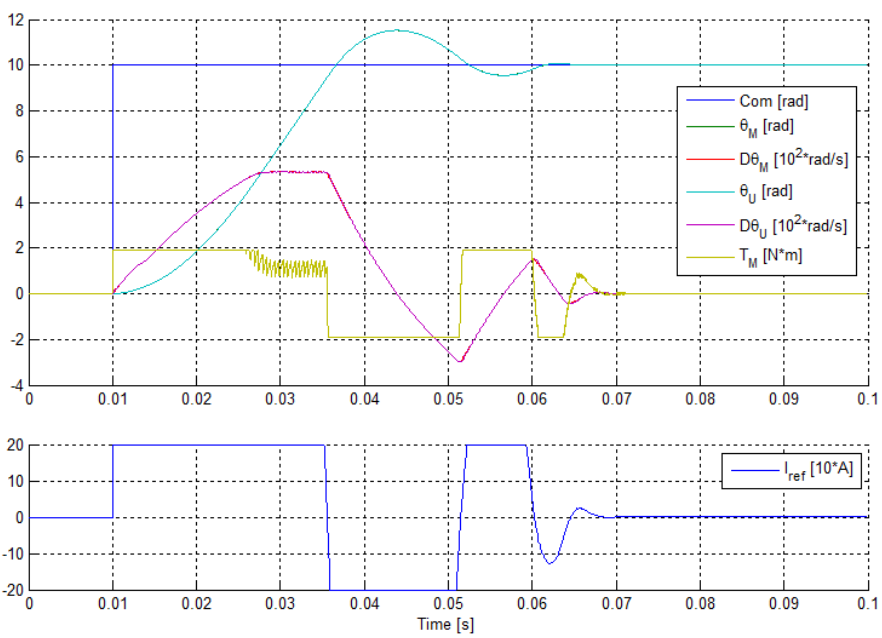

Figure 10. Example of system dynamic behavior in condition of step position command.

The ramp response analysis reveals that the proposed model is also able to simulate both a high-slope ramp response (Figure 11) and a stick-slip phenomenon (Figure 12). The first case underlines the limits of the actuator in terms of maximum speed, while the latter shows what occurs when the ramp slope is lower enough to emphasize the frictional effects. Furthermore, the model allows evaluating the incipient motion resolution of the servomechanism, i.e. the smallest command value producing an actuator's response.
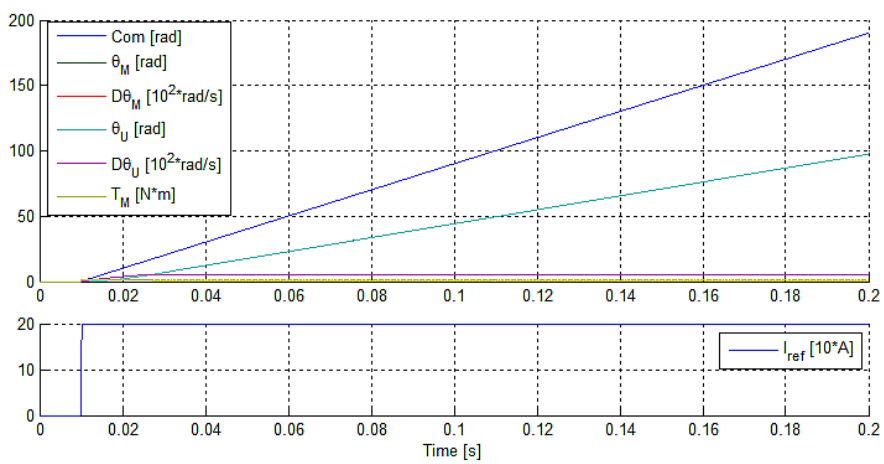

Figure 11. Example of system dynamic behavior in condition of high slope ramp command.
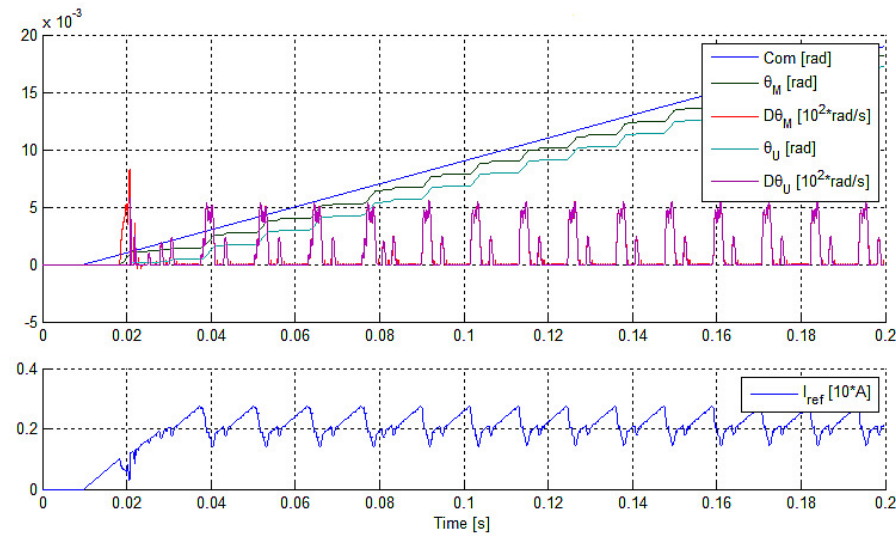

Figure 12. Example of system dynamic behavior in condition of very low slope ramp command.

At the same way, several periodic inputs have been examined confirming the model ability to simulate the behavior of the real actuation system and its sensitivity to nonlinear effects, command inputs (in terms dynamic response related to amplitude and frequency input) and external loads. The most interesting results are provided by FFT analysis performed on positions and velocities of motor and user. The algorithm employed to perform this task correctly records the most important spectral contribution of the analyzed magnitudes as a function of the corresponding command frequency. If the given command has amplitude or a frequency too wide to be properly followed by the servomechanism, this analysis also records odd multiple harmonics of the command frequency (which typically appear if signals distortions have half-wave symmetry).

\section{(b) Transmission Faults}

Checked the compliance of the proposed numerical model in NC, several analysis have been performed considering the four possible fault types. Firstly, the effects of wear conditions (friction and backlash) have been evaluated.

Frictions acting on the mechanical components of the EMA are simulated by means of a proper algorithm, proposed by Borello \& Dalla Vedova (2012). These routines are directly integrated into the corresponding Simulink dynamic models of gear motor and user in order to constitute a self-contained subsystem able to describe the effects produced by friction torques on the dynamic behavior of a generic solid mechanical moving element. In this manner, the numerical model is able to simulate correctly many of typical coulomb friction's effects as well as their interactions with the eventual mechanical ends of travel. It must be noted that in this work the considered friction torque values (referred as CFR in Figures 15-17) are always expressed as a percentage of the maximum motor torque.

The effects of the mechanical transmission backlash, according to Borello \& Dalla Vedova (2014), are simulated by the "Backlash" Simulink block (Mathworks, 2007), 
localized downstream the gear reducer (as shown in Figure 8) so as to decouple the dynamics of the gear-motor from that of the user.

The high-slope ramp command provides significant results:

1. In terms of FFT analysis on velocities, the fundamental frequency recorded in nominal conditions is around $2040 \mathrm{~Hz}$. This value slightly decreases as frictional effects increase, since angular velocity is reduced by friction. The amplitude related to this frequency monotonically increases with friction and increasing non-monotonic multiple harmonics arise (the second and the third ones have been recorded during FFT analysis). Backlash is not detected with FFT algorithm;

2. The investigation on the correlation coefficients reveals that on user position and velocity a negligible increase with friction has been found, while a definite decreasing monotonic trend can be recorded for motor torque. The same analysis performed on backlash has not provided any employable data, from a prognostic point of view. The correlation coefficient for reference current is always 1 for a ramp input, since the actuator follows a velocity regime and this fact is independent from the kind of fault implemented on the model.

Further analysis concern the sinusoidal response (the input has a frequency of $20 \mathrm{~Hz}$ and amplitude of $0.001 \mathrm{rad}$ ):

1. FFT analysis cannot detect nor friction nor backlash, since only the command frequency prevails;

2. All the correlation coefficients generally show negligible variations $(\leq 1 \%)$, regardless of changes in command frequency or amplitude. The exceptions are motor torque and reference current, which show similar monotonic, decreasing trends as friction grows: this is due to the higher torque needed to follow the command. This trend, clearer for friction and less remarked for backlash, is similar for both the wear effects.

\section{(c) BLDC Faults}

Then, the effects of BLDC faults on the performances of the servomechanism have been evaluated, considering coil short-circuit and rotor static eccentricity. These faults, according to Kim, Kim and Hur (2012), have been modeled by means of simplified numerical algorithms simulating their effects on the electrical characteristics of the BLDC motor (e.g. winding resistance, inductance and back-EMF). In particular, since both the faults affect the magnetic coupling between stator and rotor, the authors have defined an algorithm that simulates these effect modifying values and angular modulations of the back-EMF coefficients ${ }^{3}$ :

3 The proposed algorithm, implemented in the BLDC EM Model block diagram, acts on the three back-EMF constants $\mathrm{C}_{\mathrm{e}, \mathrm{i}}$ (one for each branch) modulating their trapezoidal reference values $\mathrm{K}_{\mathrm{e}, \mathrm{i}}$ as a function of coil short circuit percentage, static rotor eccentricity $\zeta$ and angular position $\vartheta_{r}$.

$$
k_{e, i}=K_{e, i} \cdot C_{e, i} \cdot\left(1+\zeta \cdot \cos \left(\vartheta_{r}\right)\right)
$$

The so obtained constants $\left(k_{e, a}, k_{e, b}, k_{e, c}\right)$ are then used to calculate the corresponding counter-electromotive forces $\left(e_{a}, e_{b}, e_{c}\right)$ and to evaluate the mechanical couples $\left(C_{e, a}\right.$, $C_{e, b}, C_{e, c}$ ) generated by the three motor phases. It must be noted that BLDC faults could be included in the simulation by modifying the values of the back-EMF constants $k_{e, i}$. In particular, the parameters of the function $f(u)$ can be changed: these values, multiplied by the effective rotor velocity $\dot{\vartheta}_{M}$, provide the corresponding real back-EMF values. A typical behavior of the system undergoing BLDC faults is the rise of sub-harmonics on the spectra of angular velocities. This phenomenon is clearly recorded with the FFT analysis on the high-slope ramp command:

1. $1 / 3$ and $2 / 3$ multiple of the fundamental harmonic are related to short-circuits; as shown in Fig. 13, the 1/3 harmonic provides the most important contribution in terms of amplitude when the fault ratio is above 0.02 ;

2. $1 / 6$ and $1 / 2$ multiple harmonics concern the rotor static eccentricity. In this case, as show in Fig. 14, the 1/6 harmonic is the prevailing term for misalignments higher than $1 \%$.

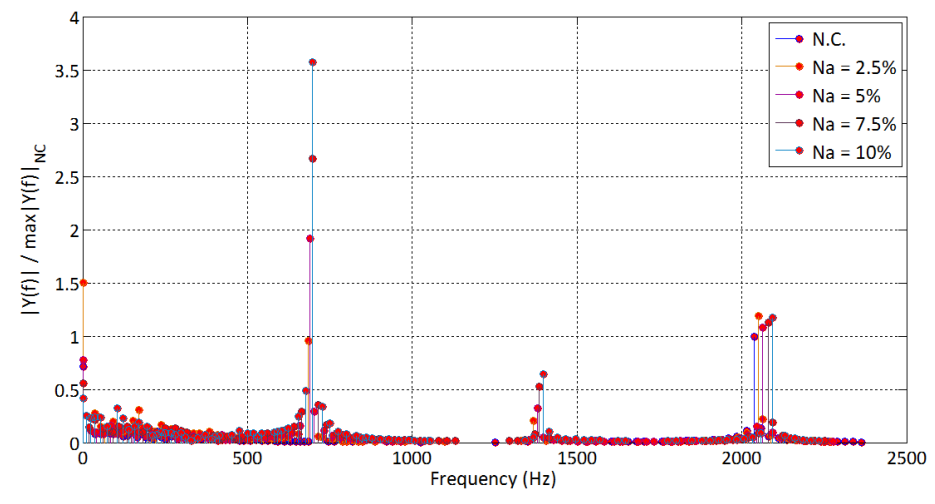

Figure 13. Rotor angular speed FFT analysis for high slope ramp command in case of progressive coil short-circuit.

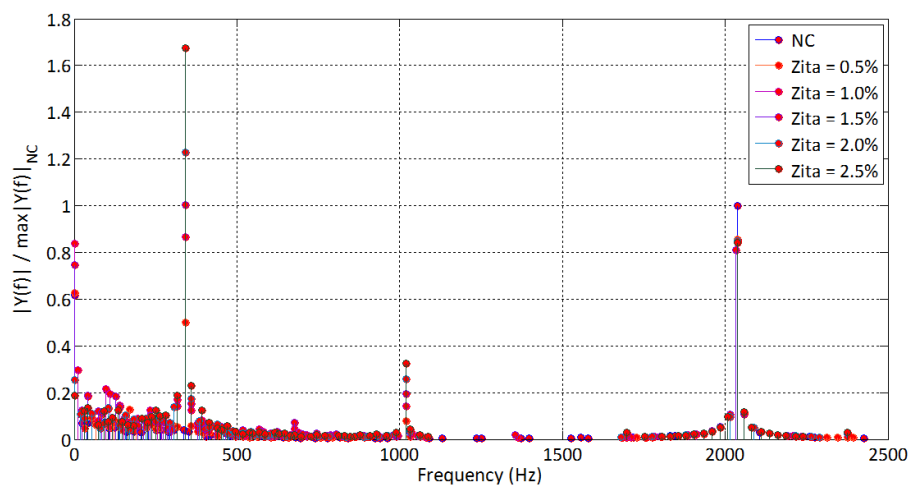

Figure 14. Rotor angular speed FFT analysis for high slope ramp command in case of progressive rotor eccentricity. 
These sub-harmonic values could be explained by means of:

$$
\frac{f_{M}}{f_{\omega, M}} \cong 2 * p * n
$$

where $f_{M}$ is the fundamental frequency recorded by FFT motor velocity analysis, $f_{\omega, M}$ is the motor velocity in $\mathrm{Hz}$, $p=4$ is the number of polar couples, $n=3$ is the number of phases. For sub-harmonics induced by coil faults, it is clear that they arise due to differences in the $n$ motor phases, so the spectral analysis detects significant contributions at $\frac{i}{n} \cdot f_{M}, i=1, \ldots, n$. The rotor static eccentricity, instead, is represented on the spectrum as combined by a subharmonic related to the number of polar couples $p$ (i.e. the $1 / 6$ sub-harmonic, for this motor) and the 1/2 sub-harmonic. The latter represents the effect of the eccentricity on a certain polar couple. In both cases, the sub-harmonic amplitudes show a monotonic trend: this result allows the detection of a possible BLDC fault with a simple observation of FFT spectra. In terms of correlation coefficient, only the motor torque shows monotonic trends for both the faults.

The sinusoidal command provides the following results:

1. As for wear detection, the FFT analysis fails due to the predominance of the command frequency;

2. Significant results are provided by the analysis on correlation coefficients $\mathrm{C}$ and a significantly decreasing monotonic trend can be recognized in reference current for progressive coil faults.

Finally, the open-loop step response has been evaluated: all the analyzed magnitudes show monotonic trends in terms of correlation coefficients for both faults, but the variations are not significant enough to be employed in prognostics.

\section{Fault Maps}

After the analysis performed on a single progressive fault, this work focuses on the effects due to the simultaneous presence of different kinds of faults acting on the system. To the purpose to achieve a timely identification and evaluation of these growing degradations, the authors developed a new faults detection/identification technique based on fault maps (FMs). A fault map constitutes the graphical representation of how a system-representative parameter varies as a function of two different types of faults. In other words, if the measurement of the real system parameter is available, this instrument allows supposing which extent a certain couple of faults has on the actuator. More exactly, a fault map displays the first fault $G_{1}$ on $\mathrm{x}$-axis and the representative parameter $P_{1}$ on y-axis. Each map represents a set of curves $P_{1}=f\left(G_{1}\right)$ that are parameterized with the second fault $G_{2}$. A proper choice of $P_{1}$ is crucial in order to obtain a useful fault map. In the first place, this parameter should be a function of both $G_{1}$ and $G_{2}$ and be highly sensitive to changes in fault levels.
In particular, its dependence from the two kinds of fault should be monotonic, i.e. the curves plotted on the maps should not intersect. The last feature is the most important, since it allows detecting a specific area on the map containing all the possible fault levels. However, the proposed prognostic technique, in order to identify the system conditions with high enough accuracy, requires more than one of these maps for a specific couple of faults. When several maps are employed, it is important that they are independent from each other. Independent maps can be obtained when the actuator undergoes different command inputs: in this way, the parameter represented on each map is a magnitude that is not related to the others. By using three independent maps, i.e. representing three different parameters $P_{1}, P_{2}$ and $P_{3}$, an accurate area containing the possible faults is identified. The considered inputs are:

1. A sinusoidal input with a frequency of $20 \mathrm{~Hz}$ and an amplitude of $0.001 \mathrm{rad}$;

2. A high-slope ramp command at $10 \mathrm{rad} / \mathrm{s}$;

3. A step command with an amplitude of $0.005 \mathrm{rad}$, with the actuator in open-loop configuration.

By using the results found during the single fault analysis to find the most suitable parameter for the map drawing, all the possible fault combinations have been investigated. It must be noted that, in many cases, the FMs were not suitable for prognostics; for few couples there were not enough independent maps (as for the couple coil short circuit - rotor static eccentricity, with only two employable maps).

A couple on which the method has been successfully tested was the friction - coil fault couple, allowing to obtain more independent maps. Among these, three were chosen to apply the FMs method ( $G_{1}=$ friction, $G_{2}=$ coil fault ratio). The first map (Figure 15) concerns correlation coefficient $C$ for reference current, $P_{1}$, obtained with sinusoidal input. The second map (Figure 16) represents the correlation coefficient $C$ for user position, $P_{2}$, when a step input is given to the open-loop system (OL). The last map (Figure 17) shows the response to a high-slope ramp input in terms of the correlation coefficient for user velocity, $P_{3}$. After the maps have been obtained, they can be employed for the proposed procedure, which is now explained in detail.

Firstly, the numerical model is simulated as affected by a known level of both friction and coil fault ratio, considering the three different command inputs: this step provides the parameters $P_{1}, P_{2}$ and $P_{3}$. As these values will be employed on the fault maps, a certain statistical dispersion, equal to $\pm 5 \%$ of the maximum variation between the curves of each map, is taken into account. Then, the first map is employed with the entering value of $P_{1}$ and an initial large area containing the possible fault levels for $G_{1}$ and $G_{2}$ is obtained. These two intervals are inserted on the second map, which requires also the value $P_{2}$ : their intersection provides narrower intervals of the two kinds of fault. 
The procedure applied on the third map (on which $P_{3}$ is considered) is the same seen for the second one. This method have been successfully employed on a number of combinations of friction and coil fault ratio, always resulting on an enough accurate detection of the fault levels acting on the actuator.

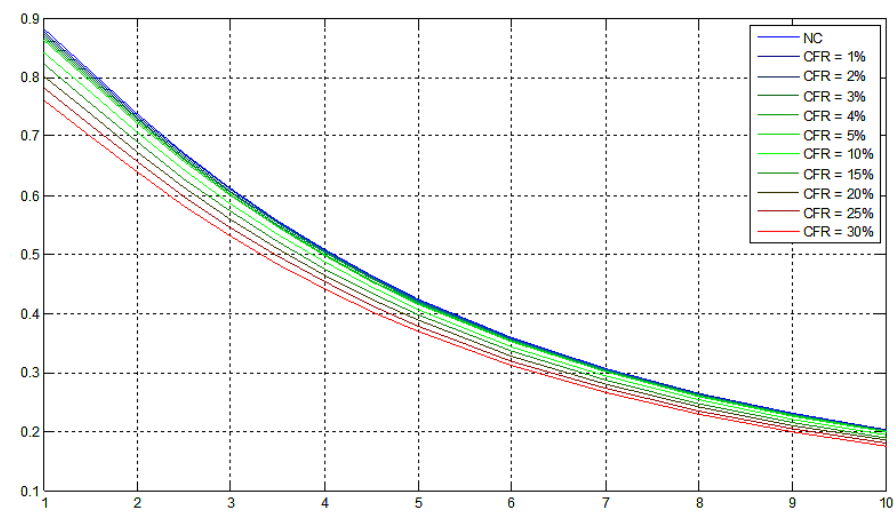

Figure 15. Correlation coefficient $\mathrm{C}$ fault map related to reference current - Sinusoidal input.

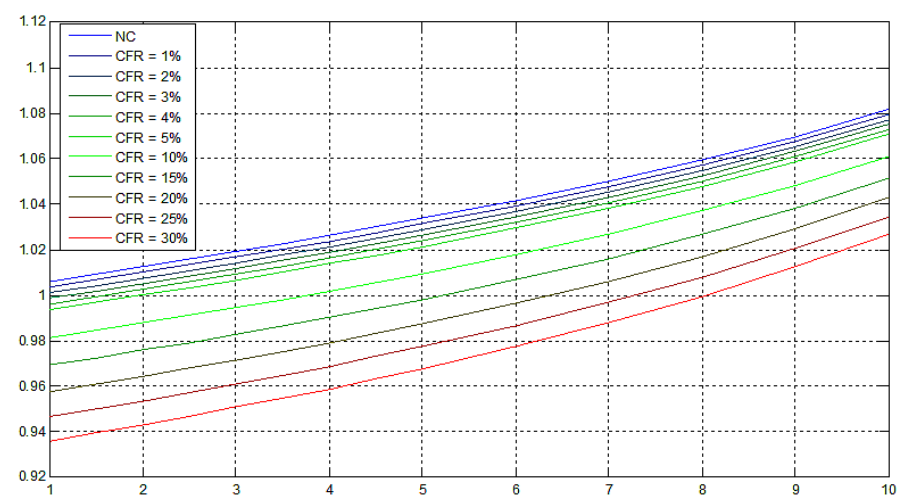

Figure 16. Correlation coefficient $\mathrm{C}$ fault map related to user position - OL Step input.

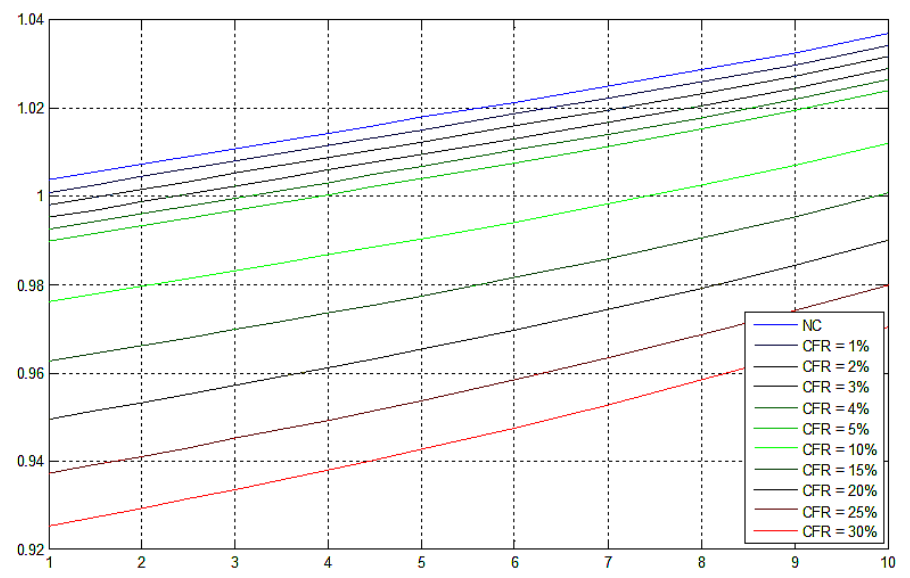

Figure 17. Correlation coefficient $\mathrm{C}$ fault map related to user velocity - High slope ramp input

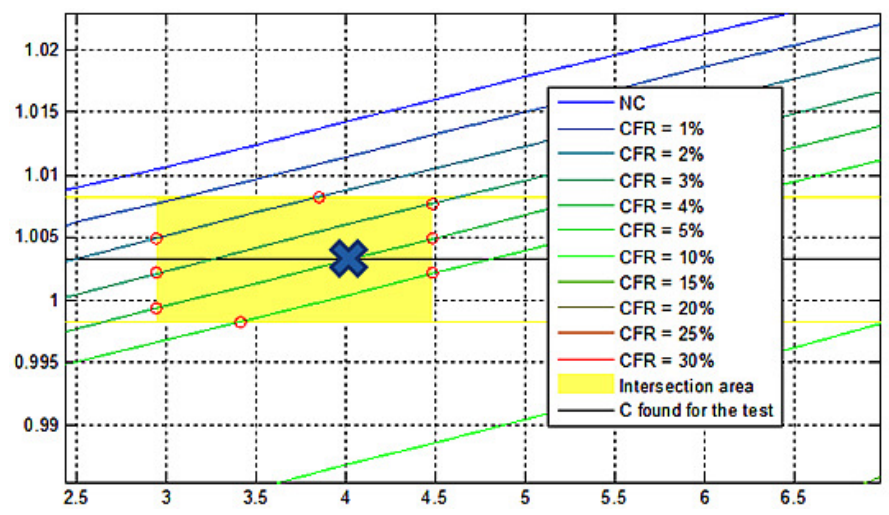

Figure 18. Example of application of Fault Maps

The example shown in Figure 18 is referring to a friction torque equal to four times the nominal value $(4 \cdot N C)$, a $4 \%$ of the coil fault ratio and a rotor static eccentricity ratio equal to 0.05 : the $X$ represented the supposed fault level. It must be noted that the correlation coefficients considered are not significantly sensitive to the variations induced in the system by low levels of backlash or rotor static eccentricity; so, the levels of friction and coil short-circuit could be properly recognized neglecting their effects.

\section{CONCLUSIONS}

This work focuses on the research of system-representative parameters, which are suitable for prognostic activities, and on the development of a technique, allowing a prompt detection of gradually increasing faults on aircraft actuators. The study has been performed on a numeric test bench (simulating a real EMA actuator) that implements four kinds of progressive faults: dry friction, mechanical backlash, turn-to-turn coil short circuit and rotor static eccentricity.

This numerical model, by proper simplifications, was then reduced, obtaining the corresponding monitoring model. The proposed fault detection/identification algorithm has been developed mixing the information derived from the spectral analysis of signals (performed by means of the FFT algorithm) and by direct comparison between EMA and monitoring model (through the correlation coefficient $C$ ). By means of these tools suitable failure precursors, useful for early recognition and quantification of the damage, have been identified. On the base of the so obtained results, proper fault maps have been drawn to perform the analysis of combined faults. This method has been successfully applied to many different combinations of considered progressive faults, guaranteeing always an enough accurate detection and estimation of their levels. 


\section{REFERENCES}

Akar, M., Taskin, S., Seker, S., \& Cankaya, I. (2010). Detection of static eccentricity for permanent magnet synchronous motors using the coherence analysis. Turkish Journal of Electrical Engineering \& Computer Science, vol. 18, n.6, pp. 963-974.

Borello, L., \& Dalla Vedova, M. D. L. (2006). Mechanical failures of flap control systems and related position errors: proposal of innovative configuration equipped with centrifugal brakes. International Journal of Mechanics and Control (JoMaC), vol. 07, n. 02, pp. 720.

Borello, L., Maggiore, P., Dalla Vedova, M. D. L., Alimhillaj, P. (2009). Dry Fiction acting on Hydraulic Motors and Control Valves: Dynamic Behavior of Flight Controls. XX National Congress AIDAA. Milan, Italy.

Borello, L., Dalla Vedova, M. D. L., Jacazio, G., \& Sorli, M. (2009). A Prognostic Model for Electrohydraulic Servovalves. Proceedings of the Annual Conference of the Prognostics and Health Management Society. September 27-October 1, San Diego. USA.

Borello, L., Maggiore, P., Villero, G., \& Dalla Vedova, M. D. L. (2010). A comparison between Dry Friction Discontinuous Computational Algorithms.27th International Congress of the Aeronautical Sciences ICAS 2010. September 19-24, Nice, France.

Borello, L., \& Dalla Vedova, M. D. L. (2012). A Dry Friction Model and Robust Computational Algorithm for Reversible or Irreversible Motion Transmission. International Journal of Mechanics and Control (JoMaC), vol. 13, n. 02, pp. 37-48, ISSN: 1590-8844.

Borello, L., \& Dalla Vedova, M. D. L. (2014). Flaps Failure and Aircraft Controllability: Developments in Asymmetry Monitoring Techniques. Journal of Mechanical Science and Technology (JMST), vol. 28, v. 11, pp. 4593-4603.

Bruzzese, C., \& Joksimovic, G. (2011). Harmonic signatures of static eccentricities in the stator voltages and in the rotor current of no-load salient-pole synchronous generators. IEEE Transactions on Industrial Electronics, vol. 58, n. 5, pp. 1606-1624.

Cardona, A., Lerusse, A., \& Géradin, M. (1998). Fast Fourier nonlinear vibration analysis. Computational Mechanics, vol. 22, n. 02, pp. 128-142.

Çunkas, M., \& Aydoğdu, O. (2010). Realization of Fuzzy Logic Controlled Brushless DC Motor Drives using Matlab/Simulink. Mathematical and Computational Applications, vol. 15, n. 02, pp. 218-229.

Dalla Vedova, M. D. L., Jacazio, G., Maggiore, P., \& Sorli, M. (2010). Identification of Precursors of Servovalves Failures for Implementation of an Effective Prognostics. International Conference of Recent Advances in Aerospace Actuation Systems and Components. May 5-7, Toulouse, France.
Ginart, A., Brown, D., Kalgren, P., \& Roemer, M. (2007). On-line Ringing Characterization as a PHM Technique for Power Drives and Electrical Machinery. Autotestcon, 2007 IEEE, September 17-20.

Ginart, A., Brown, D., Kalgren, P., \& Roemer, M. (2008). Inverter Power Drive Transistor Diagnostic and Extended Operation under One-Transistor Trigger Suppression. Applied Power Electronics Conference and Exposition, 2008. APEC 2008. February 24-28.

Gökdere, L. U., Chiu, S. L., Keller, K. J., Vian, J. (2005). Lifetime control of electromechanical actuators. IEEE Aerospace Conference Proceedings. March 5-12, Big Sky, MT. doi: 10.1109/AERO.2005.1559655

Halvaei Niasar, A., Moghbelli, H., \& Vahedi, A. (2009). Modelling, Simulation and Implementation of FourSwitch Brushless DC Motor Drive Based On Switching Functions.IEEE EUROCON 2009. May 18-23, St.Petersburg, Russia.

Haskew,T. A., Schinstock, D. E., \& Waldrep E. M. (1999). Two-Phase On" Drive Operation in a Permanent Magnet Synchronous Machine Electromechanical Actuator. IEEE Transactions on on Energy Conversion, vol. 14, n. 02.

Hemanand, T., \& Rajesh, T. (2006). Speed Control of Brushless DC Motor Drive Employing Hard Chopping PWM Technique Using DSP. Proceedings of India International Conference on Power Electronics (IICPE 2006). December 19-21, Chennai, India.

Hua, J., \& Zhiyong, H. (2008). Simulation of Sensorless Permanent Magnetic Brushless DC Motor Control System. Proceedings of the IEEE International Conference on Automation and Logistics, September, Qingdao, China.

Jeong C. L., \& Hur J. (2013). A Fast Diagnosis Technique of Inter-Turn Fault in BLDC motor using Impedance AlgorithmP. 19th International Conference on the Computation of Electromagnetic Fields. June 30 - July 4, Budapest, Hungary.

Kim, B.-W., Kim, K.-T., \& Hur, J. (2012). Simplified impedance modeling and analysis for inter-turn fault of IPM-type BLDC motor. Journal of Power Electronics, vol. 12, n. 1, pp. 10-18.

Lee, B. K., \& Ehsani, M. (2003). Advanced Simulation Model for Brushless DC Motor Drives. Electric Power Components and Systems, vol. 31, n. 9, pp. 841-868.

MathWorks (2007). Using SIMULINK Manual. The MathWorks Inc.

Shashidhara, S.M., \& Raju, P.S. (2013). Stator Winding Fault Diagnosis of Three-Phase Induction Motor by Parks Vector Approach. International Journal of Advanced Research in Electrical, Electronics and Instrumentation Engineering (IJAREIEE), vol. 2, n. 7.

Todić, I., Miloš, M., \& Pavišić, M. (2013). Position and speed control of electromechanical actuator for aerospace applications. Tehnicki Vjesnik, vol. 20, n. 5, pp. 853-860. 
Vachtsevanos, G., Lewis, F. L., Roemer, M., Hess, A., \& $\mathrm{Wu}, \mathrm{B}$. (2006). Intelligent fault diagnosis and prognosis for engineering system. Hoboken, NJ: John Wiley \& Sons, Inc.

Vichare, N., \& Pecht, M. (2006). Prognostics and Health Management of Electronics. IEEE Transactions on Components and Packaging Technologies, vol. 29, 2006, pp. 222-229.

Welch, P. D. (1967). The Use of Fast Fourier Transform for the Estimation of Power Spectra: A Method Based on Time Averaging Over Short, Modified Periodograms. IEEE Transactions on audio and electroacoustics, vol. AU-15, n. 2.

\section{BIOGRAPHIES}

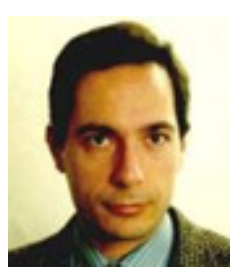

Paolo Maggiore is a professor at the Mechanical and Aerospace Engineering Department of Politecnico di Torino, that joined in 1992, where he teaches aerospace general systems engineering. Currently his students are involved in projects ranging from hydrogen fuel cell powered airplanes and UAVs, and health monitoring of flight controls, to multi-disciplinary design optimization of aerospace systems design.

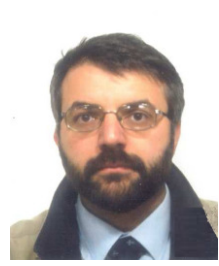

Matteo D. L. Dalla Vedova received the M.Sc. and the Ph.D. from the Politecnico di Torino in 2003 and 2007, respectively. He is currently assistant researcher at the Department of Mechanics and Aerospace Engineering. His research activity is mainly focused on the aeronautical systems engineering and, in particular, is dedicated to design, analysis and numerical simulation of on board systems and developing of prognostic algorithms for flight control systems and aerospace servomechanism.

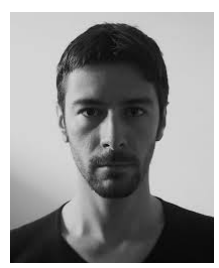

Lorenzo Pace graduated in Aerospace Engineering at Politecnico di Torino in 2008. Since 2008 to 2011, he worked as an assistant researcher, following studies about system experimental testing and modelization in the aerospace field, with a focus to energy saving techniques. Since 2012 he is completing a $\mathrm{PhD}$ in Aerospace Engineering at Politecnico di Torino, with the contribution of Thales Alenia Space, focused on the application of Model Based System Engineering to verification in the space industry.

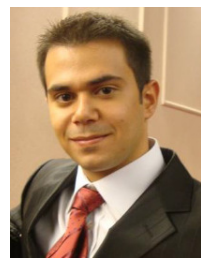

Alessio Desando graduated in Aerospace Engineering at Politecnico di Torino in 2013. His Master Degree thesis is focused on prognostic techniques for actuators. He is currently working as a research assistant from May 2013, performing studies on labyrinth seals and cyclonic separators. 